\title{
Mobile Ad Hoc Communication Issues in Ubiquitous Computing - The Smart-Its Experimentation Platforms
}

\author{
Albrecht Schmidt ${ }^{1}$, Frank Siegemund ${ }^{2}$, Michael Beigl ${ }^{4}$, Stavros Antifakos ${ }^{3}$, \\ Florian Michahelles ${ }^{3}$, and Hans-W. Gellersen ${ }^{5}$ \\ ${ }^{1}$ Media Informatics Group, University of Munich, Germany \\ albrecht.schmidtainformatik. uni-muenchen.de \\ ${ }^{2}$ Distributed Systems Group, ETH Zurich, Switzerland \\ ${ }^{3}$ Perceptual Computing and Computer Vision Group, ETH Zurich, Switzerland; \\ \{siegemund, antifakos, michahelles\}@inf.ethz.ch \\ ${ }^{4} \mathrm{TecO}$, University of Karlsruhe, Germany \\ michael@teco.edu \\ ${ }^{5}$ Computing Department, Lancaster University, UK
}

\section{European Project "Smart-Its"}

As we become familiar with the idea of connecting PDAs, notebook PCs and wallsized screens, as put forward in [1], ubiquitous computing in everyday environments creates yet new challenges for mobile ad-hoc networking. When looking at less powerful mobile and stationary devices that act as sensors or actors, new requirements for communication evolve.

\subsection{Project Vision}

The European Project "Smart-Its" [2] is interested in a far-reaching vision of computation embedded in the world. In this vision, mundane everyday artefacts become augmented as soft media, able to enter into dynamic digital relationships. In our project, we approach this vision with the development of "Smart-Its" - small-scale embedded devices that can be attached to everyday objects to augment them with sensing, perception, computation, and communication. We think of these "Smart-Its" as enabling technology for building and testing ubiquitous computing scenarios, and we use them to study emerging functionality and collective context-awareness of information artefacts.

\subsection{Project Partners}

Smart-Its is a collaboration of Lancaster University in the UK, ETH Zurich in Switzerland, University of Karlsruhe in Germany, Interactive Institute in Sweden, and VTT in Finland. The project is part of the European initiative "The Disappearing Computer", and funded in part by the Commission of the European Union, and by the Swiss Federal Office for Education and Science. 


\subsection{Wireless Communication}

A key issue in the Smart-Its project is to explore different options for wireless communication in ubiquitous computing scenarios. In this project presentation we concentrate on this aspect. In particular, we report requirements found in such environments and show ways to implement ad-hoc networks of augmented everyday objects that possess only very limited resources and computing capabilities.

\section{Ad Hoc Communication Issues in Ubiquitous Computing Environments}

From the extensive studies of settings and scenarios in wearable computing, mobile communities, and intelligent environments the different requirements for communication in ubiquitous computing have been extracted. In this section we elaborate on the most important ones.

\section{- Local Broadcast}

For many applications a mechanism that offers a broadcast to all devices in a local vicinity is important. Often, the identity of the communication partner is of minor interest; the mere fact that another object is close by is sufficient to regard it as a potential communication partner. In many cases the basic radio link offers this feature, which however is often hidden by higher protocol layers.

\section{- Radio-signal strength indication and control}

Support for adjusting transmission levels and accessing information about the received signal strength at runtime is of prime interest in many Ubicomp scenarios. These features can be used to dynamically adapt an application to the current radio environment or to limit the transmission range and thereby to control the number of potential communication partners.

\section{- Stateless vs. stateful communication}

Especially in networks where communication partners are likely to be unreliable, it is desirable that communication is not trapped in a certain state when a device fails to respond according to a certain protocol. The use of time-out mechanisms may introduce unacceptable latencies. For example, it is questionable whether connection-oriented as opposed to pure broadcast-based communication is necessary in simple Ubicomp environments.

\section{- Power issues}

For mobile computing devices, such as laptops, PDAs or mobile phones, power is of major importance. For artifacts deeply embedded in the environment, power becomes one of the most critical issues [3]. One obvious measure of power efficiency is the power expenditure per transmitted bit (unit: Joule/bit). However, this measure must not ignore the time and power spent during device discovery and connection establishment.

\section{- Instant communication}

Many applications rely on mechanisms for instant communication between devices that are in common vicinity. Therefore, it is crucial for the communication subsystem to establish relationships between devices almost instantaneously and to provide short start-up times. 


\section{- Unidirectional communication}

For particular classes of devices unidirectional communication is sufficient. In particular, simple sensing devices often only send information to other devices but never need to process any incoming messages. Whereas for simple display devices just receiving information could be desirable.

\section{- Bandwidth}

Many of the applications found in the analysis require very low bandwidth communication. In the design of a communication system bandwidth requirements of such systems should be regarded, too. In many applications it is acceptable to trade bandwidth to save energy, however, a lower bandwidth generally results in longer communication times (longer on-times) that could counter the saving effect. Therefore, in the design the optimum of bandwidth vs. energy consumption should be found.

\section{- Common Time base}

Providing a distributed common time for all participants in a network is important for distributed perception and data fusion. In general it is of particular interest to know when exactly a sensor acquired certain data.

\section{The Smart-Its Experimentation Platforms}

In the course of the Smart-Its project [2] we develop small-scale embedded devices that can be attached to everyday objects to augment them with sensing, perception, computation, and communication capabilities. The general device architecture is depicted in figure 1. Each module has an autonomous power supply, dynamically attachable and deployable sensors, a microprocessor unit, and RF-communication modules.

As communication is a vital part in the development of these devices, the design decision regarding the communication technology has far reaching implications on scenarios and applications that can be supported and built based on these devices.

To assess communication issues and to evaluate different technologies, we have developed two complementary devices; one is based on standard Bluetooth-radio technology whereas the other is based on a custom RF-communication stack.

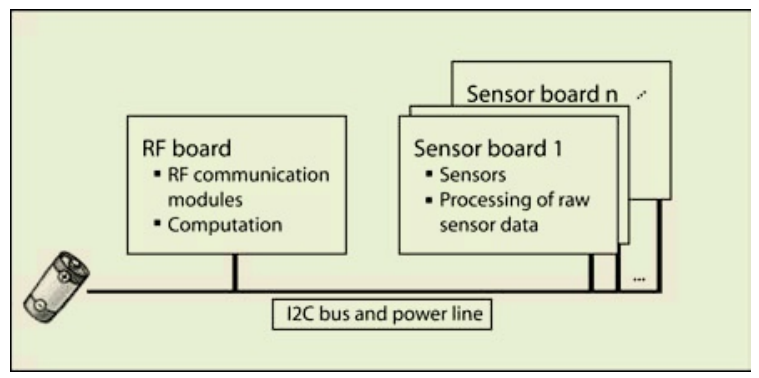

Fig. 1. Basic Smart-Its component-based device architecture. 


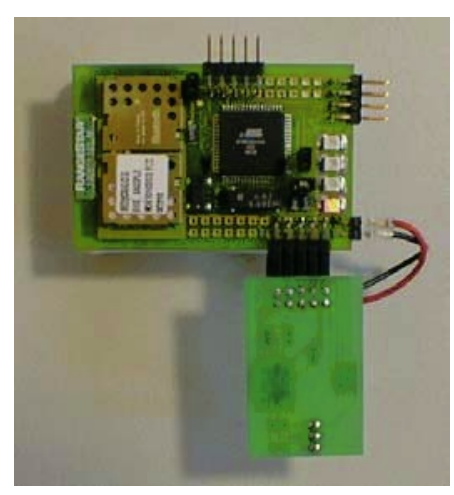

Fig. 2. Bluetooth-based Smart-It module with acceleration/ temperature sensor board attached.

\subsection{Bluetooth Smart-It}

The Bluetooth Smart-It, depicted in figure 2, is designed around an 8-bit RISC microcontroller unit with embedded memory and an Ericsson Bluetooth module [4, 5]. The modules offer a generic Host Controller Interface (HCI) to the lower layers of the Bluetooth protocol stack, while the higher layers of the protocol as well as applications must be implemented on the host system. We developed protocol stacks for some of the higher layers of the Bluetooth standard. The software supports data exchange on the L2CAP (Logical Link Control and Adaptation Protocol) and RFCOMM (the serial port emulation of Bluetooth) layers. Furthermore, objects such as calendar entries or business cards as well as commands for accessing the capabilities of mobile phones can be transmitted over standardized Bluetooth profiles that are supported by our implementation.

The main reason for choosing Bluetooth as a candidate communication technology for Smart-Its, was that it provides a degree of interoperability necessary to easily integrate augmented objects into existing computing environments. As the number of consumer devices such as PDAs, Laptops, cellular phones, and digital cameras that are equipped with Bluetooth modules increase rapidly, Bluetooth-based Smart-Its are also well suited to bridge the gap to users of smart environments by building upon the capabilities of their handheld devices. For example, Smart-Its can send business cards to mobile phones over a local Bluetooth connection to notify nearby users. Calendar entries can be stored in PDAs to remind people to maintain their objects, and SMS messages can be sent to remote entities using a nearby mobile phone as mobile access point [6].

Compared to communication protocols specifically designed for wireless sensor networks and Ubicomp environments (cf. section 3.2), communication via Bluetooth consumes significantly more energy. However, in applications where the communication modules can be switched off most of the time and the need for communication be recognized by considering local sensor readings only, the Bluetooth-based Smart-Its are well suited for realizing typical Ubicomp application scenarios. 


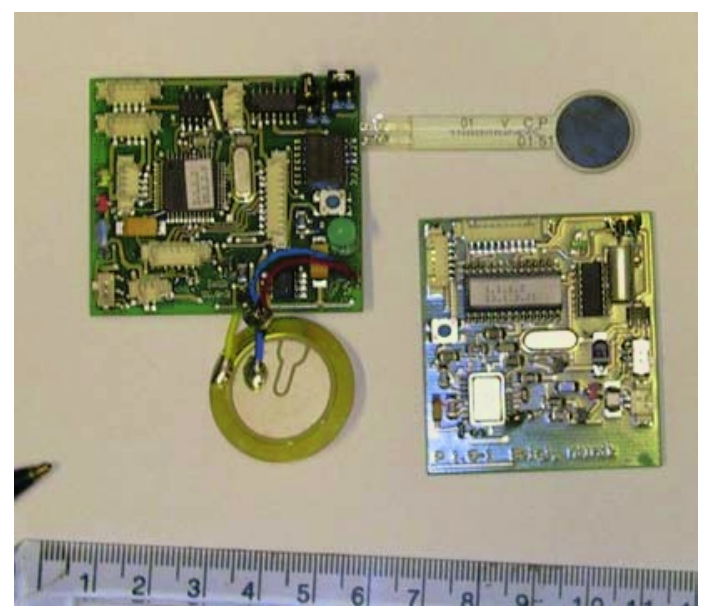

Fig. 3. On the left a fully equipped Smart-Its sensor board is shown. On the right a Smart-Its custom RF-communication board is depicted.

\subsection{Simple-RF Smart-It}

When implementing the Simple-RF Smart-It we focused on an approach that is complementary to Bluetooth (See [7] for technical details). This implementation is especially suited for applications where lowest power consumption, lower bandwidth and very fast connection times are needed. The hardware implementation is based on the TR1001 transceiver from RFM running on the European 868.35 MHz ISM band (figure 3) providing $125 \mathrm{kBit} / \mathrm{s}$ bandwidth for shared bi-directional data transfer providing a connection-to-network time of less than 12 milliseconds in average.

The main goal of this solution was to address the major requirements for an ad-hoc network intended to support communication of (small) context messages. The protocol is not designed for short range wireless multimedia communication, but specially adopted to run on very small devices with very limited amounts of energy. The RF Smart-Its protocol implements a one-hop peer-to-peer stateless datagram protocol with collision avoidance. Smart-Its broadcast information to other devices in a range of 1 to 10 meters. Physical range of the broadcast signal and therefore the resulting set of communication partners are selectable on the module for each communication packet. For addressing of communication partners different variants are available, such as addressing by the type of object (e.g. this message is for all chairs in the vicinity), by the context in which objects are in (e.g. all object that are moved within the vicinity), and also by ID based addressing.

As power consumption was one major concern in the design of the modules (as it also is for Bluetooth) several design decisions have been taken accordingly. Minimizing the time the transceiver is on and consumes energy is a key step beyond minimizing the actual power consumption of the RF-module. Based on a common time-base and transmission start time bound to a slot (transmission could start roughly every $13 \mathrm{~ms}$ in a slot of about $1 \mathrm{~ms}$ ) means that in order to listen to communication a RF-Modules has to be on only very little time (in our implementation only one millisecond within $13 \mathrm{~ms}$ which results in a reduction of the power consumption by a 
factor of about ten). This results in a run-time of up to one year with one battery set in some settings.

\section{Conclusions}

In the Smart-Its project we investigate different forms of wireless communication to suit the needs of ad-hoc communication in Ubiquitous computing environments. Analysing the requirements in such environments we explored different communication platforms - one based on Bluetooth and another based on a simple slotted protocol implemented on a microcontroller using a low power RF-Transceiver.

We realized there is no "one-fits-all" solution in this domain. In applications where interoperability is paramount and access to commercial user devices is required a Bluetooth based solution is preferable. In scenarios where energy is crucial and the amount of data communicated is small a simple RF solution has significant advantages. Also in scenarios where little information is exchanged in brief encounters (e.g. two people on bikes passing each other) it is crucial to have a system that allows rapid communication set-up, which was included in the custom RF solution.

Acknowledgement. We like to acknowledge the contributions of Oliver Kasten to the "Smart-Its"-Bluetooth platform. The work presented here was funded in part by the Commission of the European Union under contract IST-2000-25428, and by the Swiss Federal Office for Education and Science (BBW 00.0281). The project 'SmartIts' is part of the Disappearing Computer initiative, http://www.disappearingcomputer.org.

\section{References}

1. Weiser, M.: The Computer of the $21^{\text {st }}$ Century. Scientific American 265, 3, September 1991, pp. 66-75.

2. Smart-Its, EU Project. Disappearing Computer Initiative, http://www.smart-its.org. 2002.

3. Gellersen, H-W., Schmidt, A. and Beigl, M.: Multi-Sensor Context-Awareness in Mobile Devices and Smart Artefacts. ACM journal Mobile Networks and Applications (MONET), Vol. 7, No. 5. October 2002.

4. Beutel, J., Kasten, O.: A Minimal Bluetooth-Based Computing and Communication Platform. Technical Note, May 2001, http://www.inf.ethz.ch/vs/publ/

5. Kasten, Oliver, Langheinrich, Marc: First Experiences with Bluetooth in the Smart-Its Distributed Sensor Network. Workshop on Ubiquitous Computing and Communications,In: Proc. PACT 2001, Oct. 2001

6. Siegemund, F., Rohs, M.: Rendezvous Layer Protocols for Bluetooth-enabled Smart Devices, ARCS 2002, International Conference on Architecture of Computing Systems, Karlsruhe, Germany, 2002.

7. TecO, University of Karlsruhe. Smart-its Project page.

http://smart-its.teco.edu/artefacts/prototype/prototype.html. 2002. 